\title{
Are we sure to count myelinated fibers entirely? Calibration of a stereological study
}

\author{
Mehmet Emin Onger ${ }^{*}$, Stefano Geuna ${ }^{b}$, Ebru Elibol ${ }^{a}$, Aysın Pınar Turkmen ${ }^{a}$, Omur Gulsum Deniz ${ }^{a}$, Gamze Altun ${ }^{a}$, \\ Suleyman Kaplan ${ }^{\mathrm{a}}$
}

${ }^{a}$ Department of Histology and Embryology, Faculty of Medicine, Ondokuz Mayı University, Samsun, Turkey

${ }^{b}$ Department of Clinical and Biological Sciences, University of Turin, Turin, Italy

\section{ARTICLE INFO \\ * Correspondence to: \\ Mehmet Emin Önger \\ Department of Histology and Embryology, Faculty of Medicine, \\ Ondokuz Mayıs University, Samsun, Turkey e-mail: mehmetemin.onger@omu.edu.tr}

\section{Keywords: \\ Calibration \\ Peripheral nerve \\ Sampling \\ Stereology}

\section{ABSTRACT}

Peripheral nerves are subject to many different types of injuries frequently and these injuries may result in partial or total loss of motor and sensory functions. In contrast to the central nervous system, peripheral nervous system are able to undergo spontaneous regeneration. But clinical and experimental studies show that this regeneration is mostly not satisfactory. One of the most important neuropathological indicators of nerve regeneration is quantitative estimation of myelinated axons. Quantitative estimation of the total number of myelinated fibers can be obtained both by light microscopy (LM) and electron microscopy (EM) and depends on using appropriate sampling strategy. In this study, we made a direct comparison between LM and EM estimation of myelinated nerve fibers. Ten female Wistar albino rats were divided into two experimental groups; control group ( $\mathrm{n}=5)$ and gap group $(\mathrm{n}=5)$. In the gap group, $5 \mathrm{~mm}$ right sciatic nerve segment of rats were resected to form a gap. Proximal and distal nerve stumps were surrounded by collagen membrane following resection. In the control group, rats received neither any operation nor any treatment. Rats were sacrificed at 12 week after surgery. Nerve samples were embedded in resin. Semi and ultra thin sections (of $1 \mu \mathrm{m}$ and $100 \mathrm{~nm}$ thickness, respectively) were cut and stained with $1 \%$ toluidine blue (for LM) and uranyl acetate-lead citrate (for EM). Histological assessment of myelinated fibers was performed with both LM and EM using unbiased stereological counting techniques. There was a significant difference in the total number of myelinated nerve fibers between LM and EM examinations in terms of the both pure and regenerating axons. This difference is probably related to different magnification capacities in LM and EM. LM causes to underestimates as a result of possessing very small myelinated fibers, which are only detectable with EM. According to our results it may be concluded that the combination of both light and electron microscopical results is essential especially in revealing regenerating nerve fibers since very small myelinated fibers can not be distinguishable with LM.

J. Exp.Clin.Med., 2014; 31:138 